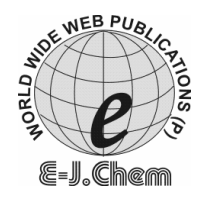

http://www.e-journals.net
ISSN: 0973-4945; CODEN ECJHAO

E-Journal of Chemistry 2010, 7(3), 882-886

\title{
Synthesis, Characterization and Antibacterial Activity of Some Halo Substituted Schiff Bases
}

\author{
S.B. JUNNE*, ARCHANA B. KADAM, \\ S.L. SHINDE ${ }^{\S}$, G.S. WAGHAMARE and Y.B. VIBHUTE. \\ P.G. Department of Chemistry, \\ Yeshwant Mahavidyalaya, Nanded-05. (M.S.), India. \\ ${ }^{\S}$ Department of Botany, Yeshwant Mahavidyalaya, Nanded, India. \\ sbjunne@gmail.com
}

Received 13 September 2009; Revised 11 November 2009; Accepted 5 January 2010

\begin{abstract}
Some new halo substituted Schiff bases have been prepared from different aromatic aldehydes and a series of substituted aromatic amines to form a number of potentially biologically active compounds. The structures of the Schiff bases have been characterized by using IR and ${ }^{1}$ HNMR spectroscopy. These compounds were screened against human pathogenic bacteria by agar diffusion method. Ampicillin was used as control.
\end{abstract}

Keywords: Aromatic amines, Aromatic aldehydes, Schiff bases, Antibacterial activity, Ampicillin.

\section{Introduction}

New halo substituted Schiff bases were used as substrates in the preparation of number of biologically active compounds ${ }^{1}$. Moreover Schiff bases were also known to have biological activities such as antimicrobial ${ }^{2-5}$, antifungal ${ }^{4-6}$, antitumor ${ }^{7-9}$ and herbicides ${ }^{10}$. They were also used as starting materials for synthesis of various biologically active heterocyclic compounds ${ }^{11}$.

Considering a literature survey of Schiff bases, we have decided to synthesize new Schiff bases which were predicted to have useful biological activity. Present study of Schiff bases have been synthesized by refluxing a mixture of different aromatic aldehydes with substituted aromatic amines and few drops of acetic acid in methanol ${ }^{12}$. On cooling, solid separated out. Solid products were crystallized from ethyl alcohol. Structures of the synthesized compounds were conformed by elemental analysis and spectral studies.

All these compounds were screened against human pathogenic bacteria i.e. Dermatophillus congolensis, Proteus vulgaris, Staphylococcus aureus, Corynebacterium 
parvum and Actinomyces bovis. The antibacterial activity was evaluated using agar diffusion method $^{13-15}$. The solvent were used as dimethyl formamide (DMF) and dimethyl sulfoxide (DMSO). Ampicillin was used as control for comparison.

$$
\mathrm{Ar}-\mathrm{NH}_{2}+\mathrm{Ar}^{\prime}-\mathrm{CHO} \quad \stackrel{\mathrm{MeOH}+\mathrm{ACOH}}{\triangle} \quad \mathrm{Ar}-\mathrm{N}=\mathrm{CH}-\mathrm{Ar}{ }^{\prime}
$$

\section{Experimental}

\section{Scheme 1}

All the melting points were taken in open capillary tube. The purity of compounds was checked by TLC using silica gel G. The IR spectra were recorded with $\mathrm{KBr}$ pellets on Perkin-Elmer 157 spectrophotometer, ${ }^{1} \mathrm{HNMR}$ spectra on Bruker WN-400 MHz FT-NMR instrument using $\mathrm{CDCl}_{3}$ on reference (Chemical shift in $\delta$, ppm).

\section{Typical procedure for synthesis of Schiff bases}

An equimolar mixture of aromatic aldehydes $(0.001 \mathrm{M})$ and substituted aromatic amines $(0.001$ M) in methanol containing acetic acid $(0.5 \mathrm{~mL})$ was refluxed for $3 \mathrm{~h}$. Excess of solvent was distilled and residue kept in cold water. The separated solid was filtered, dried and recrystallized from ethyl alcohol. The purity of the products was conformed by TLC. Details of their elemental and physical properties are given in Table 1 and spectral data are given in Table 2.

Table 1. Elemental and physical data of halo substituted Schiff bases.

Id



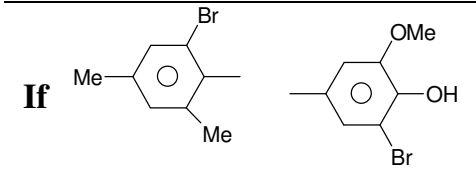

$\begin{array}{ccccccc}\mathrm{C}_{16} \mathrm{H}_{15} & 160 & 68 & 57.65 & 4.50 & 4.20 & \mathrm{Br}=24.02 \\ \mathrm{NO}_{2} \mathrm{Br} & & & (57.78) & (4.38) & (3.99) & (23.85)\end{array}$<smiles>Cc1cc(C)c([N+](=O)[O-])cc1Br</smiles><smiles>COc1cc(C)cc(Br)c1O</smiles>

$\begin{array}{cccccc}\mathrm{C}_{16} \mathrm{H}_{15} \mathrm{~N} & & & 46.48 & 3.63 & 3.38 \\ \mathrm{O}_{2} \mathrm{Br}_{2} & 110 & 72 & (46.62) & (3.78) & (3.09)\end{array}$

$\mathrm{Br}=38.74$

(39.00)

Ih<smiles>Cc1ccc(Br)cn1</smiles>

Ii<smiles>Cc1ccc(F)cc1Br</smiles><smiles>COc1cc(C)cc(Br)c1O</smiles>

$\mathrm{C}_{13} \mathrm{H}_{10} \mathrm{~N}_{2}$
$\mathrm{O}_{2} \mathrm{Br}_{2}$ $\begin{array}{lll}40.41 & 2.59 & 7.25\end{array}$ (40.10) (2.10) (7.38)

$\mathrm{Br}=41.45$

(41.28)<smiles>CC1CC=CO1</smiles>

$\mathrm{C}_{11} \mathrm{H}_{7} \mathrm{NO} \quad 55 \quad 55$

$\begin{array}{lll}49.25 & 2.61 & 5.22\end{array}$

$\mathrm{FBr}$

(48.98) (2.42) (5.01)

$\mathrm{F}=7.08$

$(6.92 \mathrm{Br}=29.85$

(30.02)

$\mathrm{Cl}=22.25$

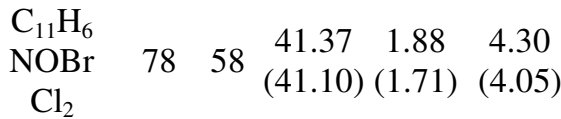

(24.89)

Table 2. IR $\left(\mathrm{cm}^{-1}\right)$ and ${ }^{1} \mathrm{HNMR}(\delta$, ppm) spectral data of halo substituted Schiff bases.

\begin{tabular}{cll}
\hline Entry & \multicolumn{1}{c}{ IR, $\mathrm{cm}^{-1}$} & \multicolumn{1}{c}{${ }^{1} \mathrm{HNMR}, \delta, \mathrm{ppm}$} \\
\hline Ia & $2920,2800\left(\mathrm{CH}_{3}\right), 1660(\mathrm{C}=\mathrm{N}), 1515$, & $3.1(\mathrm{~s}, 3 \mathrm{H}, \mathrm{OMe}), 3.2(\mathrm{~s}, 6 \mathrm{H}, \mathrm{OMe}), 8.7$ \\
& $1440\left(\mathrm{NO}_{2}\right), 1620,1600,1560(\mathrm{C}=\mathrm{C})$ & $(\mathrm{s}, 1 \mathrm{H},=\mathrm{CH}), 7.5-8.3(\mathrm{~m} .5 \mathrm{H}, \mathrm{Ar}-\mathrm{H})$. \\
Ib & $2910,2800\left(\mathrm{CH}_{3}\right), 1660(\mathrm{C}=\mathrm{N})$, & $3.0(\mathrm{~s}, 3 \mathrm{H}, \mathrm{OMe}), 3.3(\mathrm{~s}, 6 \mathrm{H}, \mathrm{OMe}) 8.8$ \\
& $1610,1600,1550(\mathrm{C}=\mathrm{C})$. & $(\mathrm{s}, 1 \mathrm{H},=\mathrm{CH}), 7.9-8.5(\mathrm{~m}, 4 \mathrm{H}, \mathrm{Ar}-\mathrm{H})$. \\
Ic & $3400(-\mathrm{OH}), 1764(\mathrm{C}=\mathrm{O}), 1655$, & $8.3(\mathrm{~s}, 1 \mathrm{H},=\mathrm{CH}), 7.5-8.1(\mathrm{~m}, 6 \mathrm{H}, \mathrm{Ar}-\mathrm{H})$, \\
& $(\mathrm{C}=\mathrm{N}), 1610,1590,1500(\mathrm{C}=\mathrm{C})$. & $11.4(\mathrm{~s}, 1 \mathrm{H}, \mathrm{OH}) 12.2(\mathrm{~s}, 1 \mathrm{H}, \mathrm{Ar}-\mathrm{OH})$. \\
Id & $3350(-\mathrm{OH}), 1670(\mathrm{C}=\mathrm{N}) 1525,1430$ & $8.8(\mathrm{~s}, 1 \mathrm{H},=\mathrm{CH}), 7.5-8.6(\mathrm{~m}, 6 \mathrm{H}, \mathrm{Ar}-\mathrm{H})$, \\
& $\left(\mathrm{NO}_{2}\right), 1610,1520,1490(\mathrm{C}=\mathrm{C})$. & $12.1(\mathrm{~s}, 1 \mathrm{H}, \mathrm{Ar}-\mathrm{OH})$. \\
Ie & $2915,2850\left(\mathrm{CH}_{3}\right), 1650(\mathrm{C}=\mathrm{N})$, & $3.2(\mathrm{~s}, 3 \mathrm{H}, \mathrm{OMe}), 8.9(\mathrm{~s}, 1 \mathrm{H},=\mathrm{CH}) 12.1$ \\
& $1620,1600,1580(\mathrm{C}=\mathrm{C}) 3320(\mathrm{OH})$. & $(\mathrm{s}, 1 \mathrm{H}, \mathrm{Ar}-\mathrm{OH}), 7.2-8.4(\mathrm{~m}, 5 \mathrm{H}, \mathrm{Ar}-\mathrm{H})$. \\
If & $2930,2845,\left(\mathrm{CH}_{3}\right), 3400(-\mathrm{OH}), 1640$ & $3.4(\mathrm{~s}, 3 \mathrm{H}, \mathrm{OMe}), 2.5(\mathrm{~s}, 6 \mathrm{H}, \mathrm{CH}), 8.8$ \\
& $(\mathrm{C}=\mathrm{N}) 1620,1590,1550,(\mathrm{Ar}-\mathrm{H})$ & $(\mathrm{s}, 1 \mathrm{H},=\mathrm{CH}), 7.5-8.4(\mathrm{~m}, 4 \mathrm{H}, \mathrm{Ar}-\mathrm{H}), 12.4$ \\
& & $(\mathrm{~s}, 1 \mathrm{H}, \mathrm{Ar}-\mathrm{OH})$ \\
Ig & $2915,2850\left(\mathrm{CH}_{3}\right), 1640(\mathrm{C}=\mathrm{N}), 3450$ & $\left.2.4(\mathrm{~s}, 6 \mathrm{H}, \mathrm{CH})_{3}\right), 3.3(\mathrm{~s}, 3 \mathrm{H}, \mathrm{OMe}), 8.7$ \\
& $(\mathrm{OH}), 1630,1595,1580(\mathrm{C}=\mathrm{C})$. & $(\mathrm{s}, 1 \mathrm{H},=\mathrm{CH}), 7.2-8.3(\mathrm{~m}, 4 \mathrm{H}, \mathrm{Ar}-\mathrm{H}), 12.2$ \\
Ih & $2930,2840\left(\mathrm{CH}_{3}\right), 3450(\mathrm{OH}), 1655$ & $(\mathrm{~s}, 1 \mathrm{H}, \mathrm{Ar}-\mathrm{OH})$. \\
& $(\mathrm{C}=\mathrm{N}), 1620,1600,1590(\mathrm{C}=\mathrm{C})$. & $(\mathrm{m}, 5 \mathrm{H}, \mathrm{Ar}-\mathrm{OM}), 12.2(\mathrm{~s}, 1 \mathrm{H}, \mathrm{OH})$. \\
Ii & $1660(\mathrm{C}=\mathrm{N}) 1625,1535,1460$ & $8.2(\mathrm{~s}, 1 \mathrm{H},=\mathrm{CH}), 6.3-7.9(\mathrm{~m}, 5 \mathrm{H}, \mathrm{Ar}-\mathrm{H})$. \\
& $(\mathrm{C}=\mathrm{C}), 1050(\mathrm{C}-\mathrm{O}-\mathrm{C})$. & \\
Ij & $1665(\mathrm{C}=\mathrm{N}), 1630,1535,1490$, & $8.2(\mathrm{~s}, 1 \mathrm{H},=\mathrm{CH}), 6.3-7.9(\mathrm{~m}, 5 \mathrm{H}, \mathrm{Ar}-\mathrm{H})$. \\
& $(\mathrm{C}=\mathrm{C}), 1020,(\mathrm{C}-\mathrm{O}-\mathrm{C})$. & \\
& &
\end{tabular}




\section{Assessment of antibacterial activity of Schiff bases}

The antibacterial test was performed using the agar diffusion method of Collins et al ${ }^{16}$. The test organisms were inoculated on nutrient agar plates and spread uniformly using a sterile glass spreader. Wells of $5 \mathrm{~mm}$ in diameter were made on the nutrient agar using a sterile cork borer. To each wells, the different concentration of different compound was introduced. Ampicillin was used as control. The plates were incubated at $37{ }^{\circ} \mathrm{C}$ for $24 \mathrm{~h}$. The zones of inhibition were then recorded.

\section{Determination of minimum inhibitory concentration (MIC) of extracts}

The MIC of these compounds were determined on solid medium (Nutrient agar) using the method of Collins et $a l^{16}$. The range of concentration was used 3.5 to $6.5 \mathrm{mg} / \mathrm{mL}$.

\section{Results and Discussion}

Aromatic aldehydes and substituted aromatic amines were chosen as the starting materials. Treatment of different aromatic aldehydes with aromatic amines in solvent methanol gave the desired Schiff bases. These Schiff bases shows IR absorption peak at $1660-1635 \mathrm{~cm}^{-1}(\mathrm{C}=\mathrm{N}$ stretching). Antibacterial activity recorded in terms of average zones of inhibition in millimeter $(\mathrm{mm}$.) in Table 3 . These compounds showed a range of activity against most of the tested bacteria. The dimethyl formamide (DMF) and dimethyl sulfoxide (DMSO) solution exhibited very strong activity against two bacteria i.e., Dermatophillus cangolensis and staphylococcus aureus and only one compound in DMF solution failed to exhibit any significant antibacterial activity i.e. Ig for Proteus vulgaris and Staphylococcus aureus where as in DMSO solution Ic and Id also failed to exhibit antibacterial activity against Actinomyces bovis. Most of the compounds showed more activity than control Amphicillin. The MIC (Minimum inhibitory concentration) of these compounds in both solvent against the test organisms ranged between 3.5 to $6.5 \mathrm{mg} / \mathrm{mL}$.

Table 3. Antibacterial activity of halo substituted Schiff bases.

\begin{tabular}{|c|c|c|c|c|c|c|c|c|c|c|c|}
\hline \multirow{2}{*}{ S. No. } & \multirow{2}{*}{ Entry } & \multicolumn{5}{|c|}{ DMF } & \multicolumn{5}{|c|}{ DMSO } \\
\hline & & $D c$. & $P v$. & Sa. & $C p$. & $A b$. & $D c$. & $P v$. & $S a$. & $C p$. & $A b$. \\
\hline 1 & Ia & +++ & ++ & ++ & ++ & +++ & +++ & ++ & +++ & + & + \\
\hline 2 & Ib & ++++ & ++++ & ++++ & ++++ & ++++ & ++++ & ++++ & ++++ & ++ & ++ \\
\hline 3 & Ic & ++++ & + & ++ & + & + & ++++ & + & ++++ & ++ & - \\
\hline 4 & Id & ++ & ++ & +++ & ++ & ++ & ++ & ++ & + & +++ & - \\
\hline 5 & Ie & ++++ & ++++ & ++++ & ++ & +++ & ++++ & ++++ & +++ & ++++ & ++++ \\
\hline 6 & If & +++ & + & + & + & + & ++++ & +++ & +++ & ++ & + \\
\hline 7 & Ig & +++ & - & - & ++ & ++ & ++++ & ++ & ++++ & + & + \\
\hline 8 & Ih & +++ & + & ++ & ++ & +++ & ++++ & + & +++ & + & + \\
\hline 9 & Ii & +++ & +++ & +++ & +++ & ++ & ++++ & +++ & +++ & +++ & ++ \\
\hline 10 & $\mathbf{I j}$ & +++ & ++ & +++ & + & ++ & +++ & ++ & ++++ & + & + \\
\hline Control & Ampicillin & +++ & +++ & +++ & ++ & +++ & +++ & ++ & +++ & ++ & ++ \\
\hline
\end{tabular}

\# DMF = Dimethyl formamide, \# DMSO=Dimethyl sulfoxide, \# Dc= Dermatophilus congolensis, \# Pv =Proteus vulgaris, \# Sa= Staphylococcus aureus, \# Cp= Corynebacterium parvum. \# Ab= Actinomyces bovis. $=$ No inhibition, $+=5-10 \mathrm{~mm}$ diameter of zone of inhibition.$++=10-15 \mathrm{~mm}$ diameter of zone of inhibition. $+++=15-20 \mathrm{~mm}$. diameter of zone of inhibition. $++++=20-25 \mathrm{~mm}$ diameter of zone of inhibition. $+++++=25-30 \mathrm{~mm}$ diameter of zone of inhibition. 


\section{Acknowledgments}

The authors are thankful to Principal, Yeshwant Mahavidyalaya, Nanded for providing laboratory facilities and the Director of IICT, Hyderabad for providing IR, ${ }^{1} \mathrm{HNMR}$ spectra. The authors are also thankful to Western Regional office U.G.C, Pune for providing financial support (Project file no.47-876/09).

\section{References}

1. Karia F D and Parsania P H, Asian J Chem., 1999, 11, 991-995.

2. More P G, Bhalvankar R B and Pattar S C, J Indian Chem Soc., 2001, 78, 474-475.

3. El-Masry A H, Fahmy H H and Abdelwahed S H A, Molecules, 2000, 5, 1429-1438.

4. Baseer M A, Jadhav V D, Phule R M and Vibhute A Y, Oriental J Chem., 2000, 16, 553-556.

5. Pandeya S N, Sriram D, Nath G and De clerca E, I L Farmaco, 1999, 54, 624-628.

6. $\quad$ Singh W M and Dash B C, Pesticides, 1988, 22, 33-37.

7. Hodnett E M and Dunn W, J Med Chem., 197, 13, 768-770.

8. Desai S B, Deai P B and Desai K R, Heterocycl Commun., 2001, 7, 83-90.

9. Pathak P, Jolly V S and Sharma K P, Oriental J Chem., 2000, 16, 161-162.

10. Samadhiya S. and Halve A, Oriental J Chem., 2001, 17,119-122.

11. Junne S B, Wadje S S, Baig M M V and Vibhute Y B, Int J Chem Sci., 2007, 5(5), 2093-2101.

12. Sridhar S K, Saravanan M and Ramesh A, Eur J Med Chem., 2001, 36, 615.

13. Reddy Thirupathi Y and Reddy Narsimha P, Indian J Chem., 2005, 44B, 1080.

14. Collins C H, Microbiological Methods, Butterworth, London, 1967, 364.

15. Baig M M V, Role of cell wall degrading enzymes induction of resistance in plants, Ph.D. Thesis, S.R.T.M. University, Nanded, 1977.

16. Collins C H, Lynes P M and Grange J M, Microbiological Methods, $7^{\text {th }}$ Edition Butterwort-Heinemann Ltd., Britain, 1995, 175-190. 


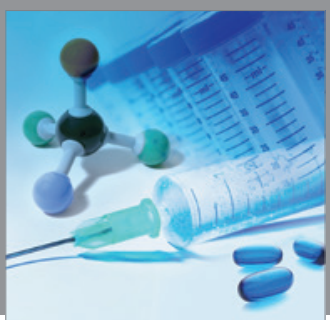

International Journal of

Medicinal Chemistry

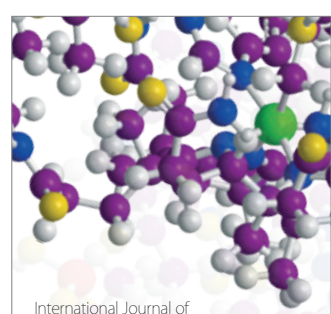

Carbohydrate Chemistry

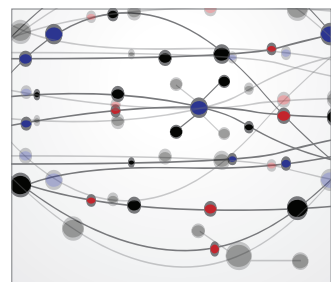

The Scientific World Journal
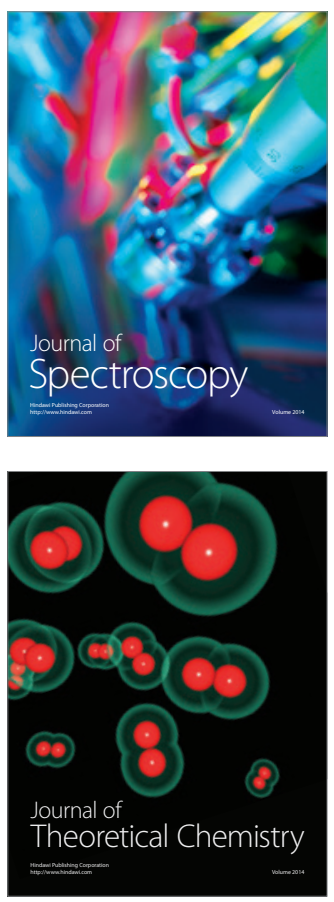
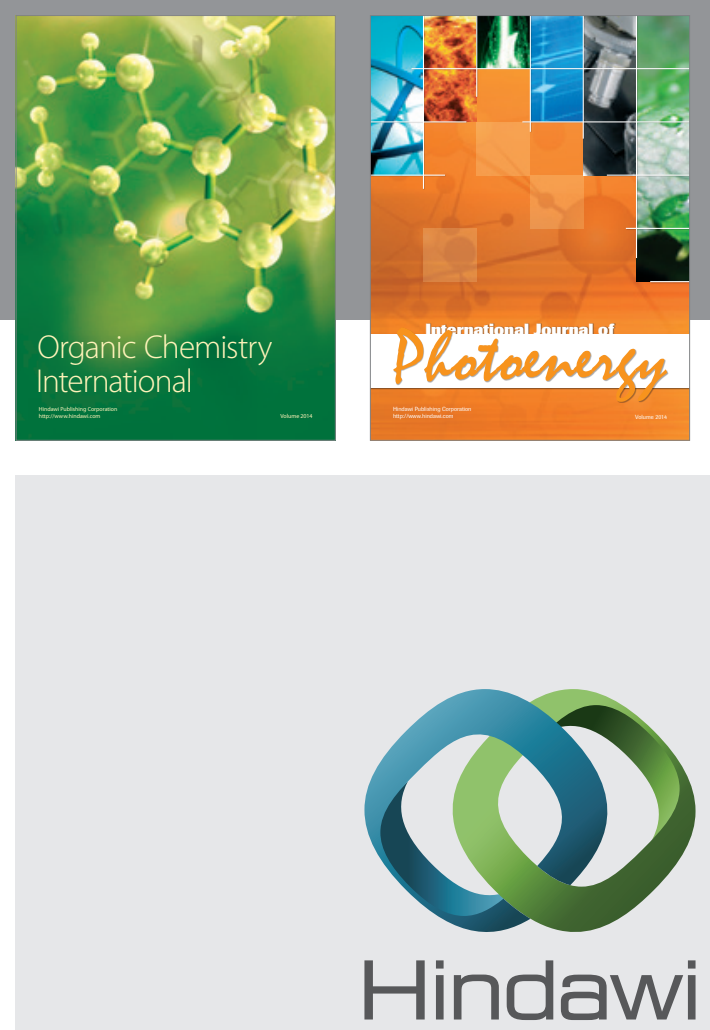

Submit your manuscripts at

http://www.hindawi.com
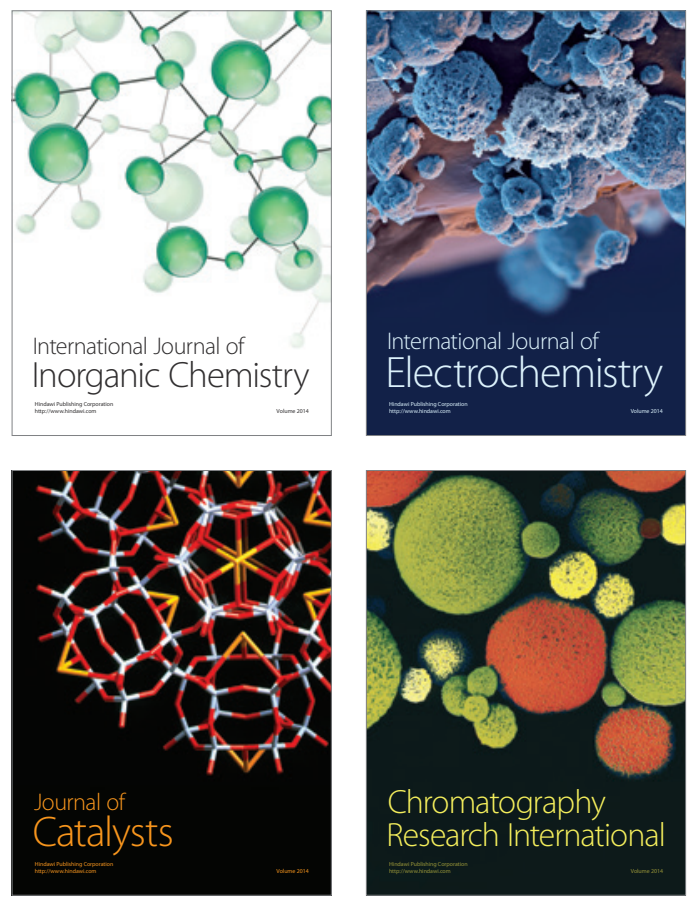
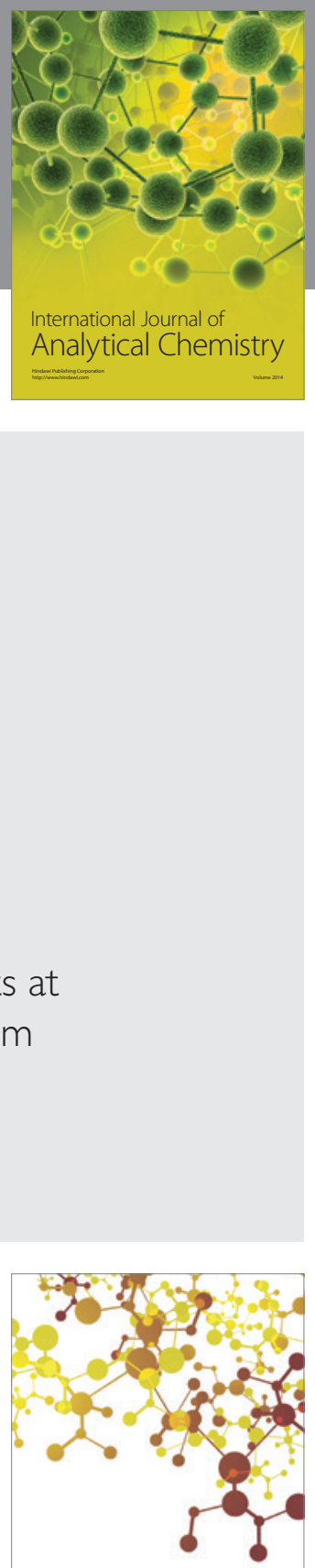

Journal of

Applied Chemistry
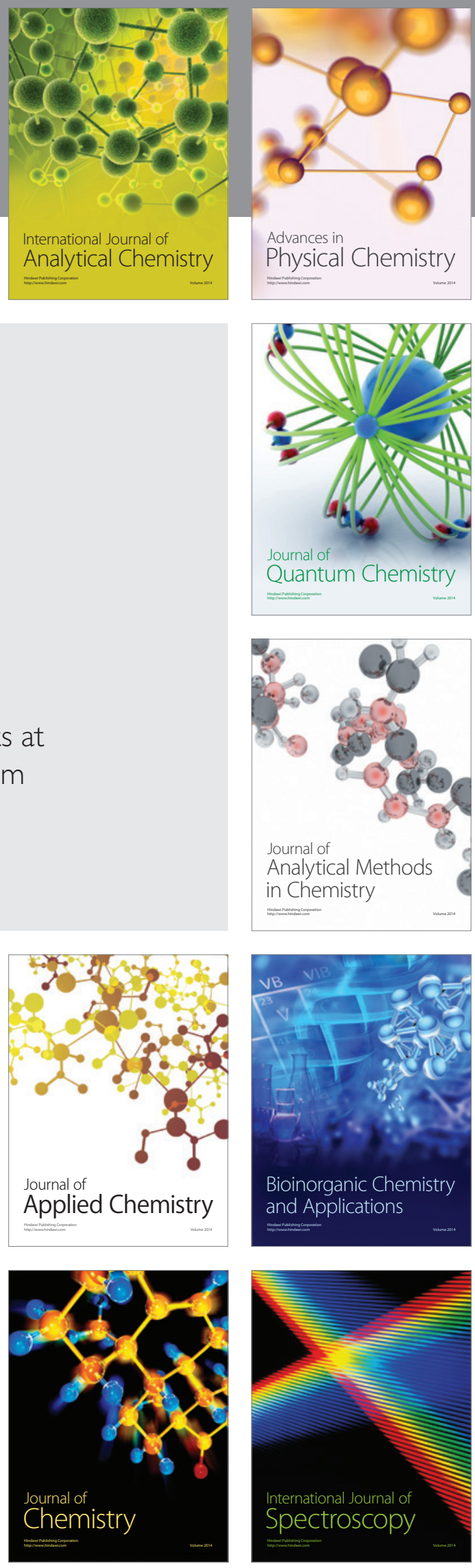